\title{
The influence of maternal glucose metabolism on fetal growth, development and morbidity in 917 singleton pregnancies in nondiabetic women
}

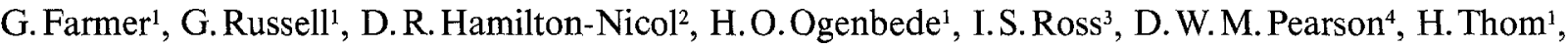 \\ D.F. Kerridge ${ }^{5}$ and H.W. Sutherland ${ }^{2}$
}

Departments of ${ }^{1}$ Child Health, ${ }^{2}$ Obstetrics and Gynaecology, ${ }^{3}$ Chemical Pathology, ${ }^{4}$ Medicine and ${ }^{5}$ Statistics, University of Aberdeen, Aberdeen, Scotland, UK

\begin{abstract}
Summary. To study the effects on the fetus of variations in maternal glucose tolerance, a $25 \mathrm{~g}$ rapid intravenous glucose tolerance test was performed at or about 32 weeks gestation in 917 randomly selected nondiabetic women with singleton pregnancies. The results were withheld from the patients and their obstetricians and paediatricians, and no treatment or advice was offered. Fasting plasma glucose and indices of glucose disposal (including a new index which we have termed "summed glucose") were distributed unimodally, with no evidence of a separate pathological group towards the diabetic end of the distributions. Significant associations were found between maternal glucose metabolism and var-
\end{abstract}

ious measures of neonatal nutrition and morbidity, including the incidence of congenital malformations and morbidity related to asphyxia, suggesting that variations within the normal range in maternal glucose metabolism can influence growth and development in the fetus. These relationships were continuous throughout the range of maternal glucose tolerance and were not of predictive value in individual cases.

Key words: Pregnancy, glucose tolerance test, blood glucose, infant, newborn, birth weight, asphyxia neonatorum, abnormalities.
Although diminished glucose tolerance during pregnancy is associated with increased birthweight and increased neonatal morbidity, including an increased incidence of congenital malformations $[1,2]$ it is not known if there is an identifiable degree of maternal glucose intolerance or of fasting hyperglycaemia beyond which fetal well-being is predictably compromised, nor if in the nondiabetic pregnant woman the disposal of intravenously administered glucose is distributed unimodally, or bimodally due to the presence of a pathological group with "impaired glucose tolerance".

The present study of a pregnant population unselected except for the exclusion of diabetic women and twin pregnancies was designed to investigate the relationships between maternal glucose metabolism and fetal outcome, and to define the distribution of normal values during pregnancy for fasting plasma glucose (FPG) and glucose tolerance as assessed by the intravenous glucose tolerance test. The intravenous test was chosen for this study because it gives results which reflect glucose disposal in numerical indices $[3,4]$ which have been applied widely during pregnancy [3,5-12] and which are readily applicable to epidemiological research

\section{Subjects and methods}

\section{Ethical considerations}

Before the study was started, a detailed protocol was submitted to and approved by the Joint Ethical Committee of Grampian Health Board and the University of Aberdeen.

\section{Patients studied}

Of 1848 Caucasian women who were approached at their initial visit to the antenatal clinic at Aberdeen Maternity Hospital, the only obstetrical facility in the city of Aberdeen, 937 consented to participate in the study. After excluding 10 twin pregnancies and 10 patients who for various other reasons failed to comply with the study protocol, 917 patients finally attended for an intravenous glucose tolerance test at as near to 32 weeks gestation as practicable. With the agreement of the entire specialist staff of the Hospital, the results of the tests were not made available to the patient nor to the responsible obstetrician or paediatrician and therefore, could not influence maternal diet or the clinical management of the mother or her baby.

Although no prospective attempt was made to ensure that the mothers approached were representative, the women who participated in the study were broadly similar to the population from which they were drawn (i. e. all patients from the city of Aberdeen delivering in Aberdeen Maternity Hospital during the study period) in terms of maternal age, parity, mode of delivery and perinatal mortality after the 32 nd week of pregnancy (i.e. after the gestation when the olucose tolerance test was performed). There was a slight over- 
representation of upper socio-economic groups in the study group, inevitable in a study requiring patient interest, time and cooperation.

\section{Maternal clinical data}

Height was measured at the first antenatal visit. Weight was measured at each visit in a standard hospital gown and, where necessary, weight at 20 weeks gestation (for use in statistical analyses involving maternal weight) was calculated by extrapolation. Socio-economic group was determined as far as possible by the husband's or putative father's occupation [13].

\section{Assessment of maternal glucose metabolism}

The technique of glucose tolerance testing was identical to that described previously [10]; the rate of glucose disposal during the first hour after the intravenous administration over $3 \mathrm{~min}$ of $25 \mathrm{~g}$ glucose was expressed as the rate of fall of the difference between actual capillary plasma glucose and FPG (Increment Index, II) [14] and as the rate of fall of the absolute glucose concentration (Absolute Index, AI) [4]. Impaired glucose tolerance is therefore reflected in low values for II and $A I$.

All available plasma glucose results are used in the calculation of AI. Because plasma glucose commonly falls below the fasting level resulting in a "negative increment" which renders log transformation impossible (noted in $35 \%$ of cases in the present series), and because log transformation of small numbers magnifies scatter, II is calculated only from that part of the curve in which all plasma glucose values exceed an arbitrary value of $1.1 \mathrm{mmol} / 1$ above fasting.

$\mathrm{AI}$ and II were calculated both graphically [8] and by the least squares method. The Pearson correlation coefficient between II calculated by least squares and II calculated graphically was 0.99 ; the mean difference between the two values was $0.01 \%$ per min (SD 0.2). The corresponding values for AI were 0.98 and $0.25 \%$ per min (SD 0.08). All further data are based on graphical calculation of the two indices.

Where available, the sum of all the glucose values, including the FPG but excluding the $4 \mathrm{~min}$ value, has been used to approximate the area under the intravenous glucose tolerance test (IVGTT) curve; we have termed this index the "summed glucose".

To assess maternal insulin response to injected glucose [15], immediately following completion of the injection of glucose (i.e. $3 \mathrm{~min}$ after the start of the injection) blood was drawn from the contralateral arm and plasma separated and stored at $-20^{\circ} \mathrm{C}$ for subsequent batch analysis of insulin by radioimmunoassay.

\section{Assessment of neonatal nutrition}

Gestational age was calculated from the date of the first day of the last menstruation supplemented in cases where the menstrual data were considered unreliable by ultrasound scan in early pregnancy. Birthweight was measured unclad within the first $2 \mathrm{~h}$ of life. Birthweight standard deviation score [16] was calculated from gestational age, birth rank and sex but was not corrected for maternal stature. Mid-thigh skinfold thickness, was measured using Holtain calipers (Holtain Ltd, Crosswell, Crymmych, Dyfed, UK) and corrected for sex and birthweight [17]. Length was measured with a calibrated measuring board and occipitofrontal circumference with a disposable paper tape measure.

\section{Assessment of potential neonatal morbidity}

Potential neonatal morbidity, identified by review of the clinical records, was classified under the following headings:

Congenital anomalies. Minor (deviations from normal requiring no treatment and causing no inconvenience). Moderate (producing significant inconvenience or disability or requiring treatment). Severe (causing major handicap or death). Disorders transmitted by simple Mendelian inheritance (e.g. sickle cell disease, haemophilia) were excluded from the statistical analysis of congenital anomalies; an infant with arthrogryposis multiplex congenita, a disorder which, although sometimes inherited, is more often sporadic, was included in the statistical analysis.

Jaundice. Serum bilirubin in excess of $250 \mu \mathrm{mol} / 1$. Two infants who were stillborn and a third infant who died during the first hour of life were excluded from analysis of jaundice.

Transient tachypnoea. Self-limiting respiratory distress of more than $6 \mathrm{~h}$ duration; specific diagnoses such as hyaline membrane disease, sepsis and pneumothorax were excluded.

Low plasma glucose. Laboratory plasma glucose $<2 \mathrm{mmol} / 1$, either on sample taken at $1 \mathrm{~h}$ of age as part of study protocol, or subsequently on sample taken for clinical reasons; this value was chosen as it is used locally as the criterion for incipient neonatal hypoglycaemia.

Sepsis. Minor (superficial lesions which, in the opinion of the attending paediatrician, did not require systemic antibiotic treatment). Suspected severe (antibiotics administered on a speculative basis because of strong clinical suspicion of sepsis, sepsis not subsequently proven on culture). Severe (confirmed diagnosis of systemic infection).

Birth asphyxia. Apgar score $<4$ at $1 \mathrm{~min}$ or $<7$ at $5 \mathrm{~min}$, and/or laboratory evidence of severe intrapartum asphyxia (base excess < $-11 \mathrm{mmol} / 1$ at age $1 \mathrm{~h}$ ).

Morbidity. The presence of any of the above.

Admission to special care baby unit. Any admission regardless of reason or duration.

\section{Statistical analysis}

The data were analysed using the Statistical Package for Social Sciences [18]. Missing data, particularly skinfold measurements which were performed only if one specified observer was available, are presumed to have occurred randomly, and we have analysed all available data, rather than restricting our analyses to those cases in which data were complete; numbers available for analysis are shown in parentheses in the Tables. The distributions of FPG, AI and II were appreciably skew and a natural logarithmic transformation was therefore applied prior to statistical analysis. Ninety-five percent confidence intervals were calculated using the method described by Gardner and Altman [19], and are presented graphically.

\section{Results}

The relationships between the indices of maternal glucose metabolism together with other factors which might be expected to influence neonatal nutrition, and various neonatal measurements are presented as Pearson correlation coefficients in Table 1 . It can be seen that there are numerous statistically significant correlations between the indices of maternal glucose metabolism and infant size. AI correlates more consistently with infant size than any of the other maternal variables, but in general the correlation coefficients are of low magnitude and attain statistical significance only through the large number of cases studied. The stron- 
Table 1. Pearson correlation coefficients $(r)$ between indices of maternal glucose metabolism, logged $\left(l_{n}\right)$ where appropriate, and indices of neonatal growth and nutrition. ( $n=$ number of cases available for analysis). SDS-Standard deviation score

\begin{tabular}{lllcccc}
\hline & & $1_{\mathrm{n}} \mathrm{FPG}$ & \multicolumn{1}{c}{$\mathrm{1}_{\mathrm{n}} \mathrm{II}$} & $1_{\mathrm{n}} \mathrm{AI}$ & $\begin{array}{l}\text { Summed } \\
\text { glucose }\end{array}$ & $\begin{array}{l}\text { Insulin } \\
\text { response }\end{array}$ \\
\hline Birth- & $r$ & 0.0982 & -0.1156 & -0.2819 & 0.0165 & -0.0608 \\
weight & $(n)$ & $(917)$ & $(917)$ & $(917)$ & $(868)$ & $(864)$ \\
SDS & $p$ & 0.001 & $<0.001$ & $<0.001$ & 0.313 & 0.037 \\
Thigh & $r$ & 0.1991 & -0.0677 & -0.2110 & 0.0781 & 0.0429 \\
skin- & $(n)$ & $(751)$ & $(751)$ & $(751)$ & $(712)$ & $(709)$ \\
fold & $p$ & $<0.001$ & 0.032 & $<0.001$ & 0.019 & 0.127 \\
Corrected & $r$ & 0.1693 & -0.0313 & -0.0918 & 0.1203 & 0.1042 \\
skinfold & $(n)$ & $(751)$ & $(751)$ & $(751)$ & $(712)$ & $(709)$ \\
thickness & $p$ & $<0.001$ & 0.196 & 0.006 & 0.001 & 0.003 \\
Crown- & $r$ & 0.0771 & -0.0357 & -0.1517 & -0.0460 & -0.0926 \\
heel & $(n)$ & $(916)$ & $(916)$ & $(916)$ & $(867)$ & $(863)$ \\
length & $p$ & 0.010 & 0.140 & $<0.001$ & 0.008 & 0.003 \\
Crown- & $r$ & 0.0711 & -0.0610 & -0.1755 & -0.0250 & -0.0698 \\
rump & $(n)$ & $(914)$ & $(914)$ & $(914)$ & $(865)$ & $(861)$ \\
length & $p$ & 0.016 & 0.033 & $<0.001$ & 0.232 & 0.020 \\
Occipito- & $r$ & 0.0549 & -0.0526 & -0.1727 & -0.0204 & -0.0811 \\
frontal & $(n)$ & $(916)$ & $(916)$ & $(916)$ & $(867)$ & $(863)$ \\
circum- & $p$ & 0.048 & 0.056 & $<0.001$ & 0.275 & 0.009 \\
ference & & & & & & \\
\hline
\end{tabular}

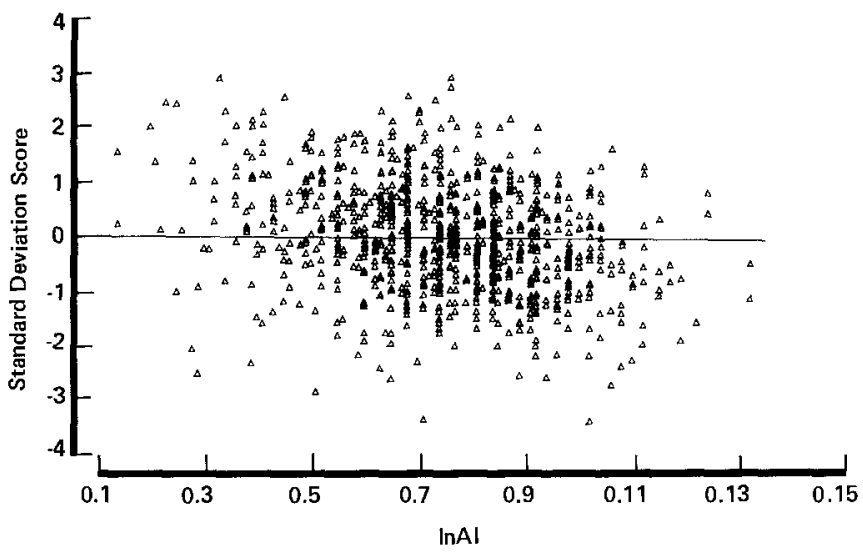

Fig. 1. Relationship between log maternal absolute index $\left(1_{n} A I\right)$ and infant birthweight standard deviation score (SDS)

gest of these associations, that between maternal AI and birthweight standard deviation score (SDS), is shown graphically in Figure 1. It is clear from this figure that birthweight SDS varies with AI across its entire range; there is no evidence of a "pathological" group at the "diabetic" end of the spectrum. Similar features were noted when the other correlations were examined graphically.

Because maternal height, weight, age and parity are mutually interdependent variables, partial correlation coefficients relating these variables to the indices of maternal glucose metabolism and to neonatal nutrition were calculated; no significant change in the pattern of correlation was found.

The differences in the mean values of the maternal glucose tolerance indices between groups where specific forms of potential or actual morbidity were "pre-
Table 2. Numbers of cases analysed in the preparation of $95 \%$ confidence intervals presented in Figures 3 and 5

\begin{tabular}{|c|c|c|c|}
\hline & $\begin{array}{l}1_{n} \mathrm{AI} \\
1_{n} \mathrm{AI} \\
1_{n} \mathrm{FPG}\end{array}$ & $\begin{array}{l}\text { Summed } \\
\text { plasma } \\
\text { glucose }\end{array}$ & $\begin{array}{l}\text { Insulin } \\
\text { response }\end{array}$ \\
\hline ongenital anomaly mild (all & $94: 793$ & $89: 750$ & $88: 746$ \\
\hline moder & $29:$ & $28: 750$ & $29: 746$ \\
\hline Transient tachypnoea (all cases) & $38: 870$ & $34: 827$ & $38: 818$ \\
\hline gestation $<41$ weeks & $32: 614$ & $29: 581$ & $32: 576$ \\
\hline gestation $\geq 41$ weeks & $6: 256$ & $5: 246$ & $6: 242$ \\
\hline Hypoglycaemia (all cases) & $154: 763$ & $145: 723$ & $114: 720$ \\
\hline Jaundice (all cases) & $85: 829$ & $77: 788$ & $76: 785$ \\
\hline Sepsis minor (all cases) & $13: 810$ & $12: 769$ & $11: 763$ \\
\hline suspected severe (all cases) & $84: 810$ & $79: 769$ & $81: 763$ \\
\hline severe (all cases) & $10: 810$ & $8: 769$ & $9: 763$ \\
\hline \multicolumn{4}{|l|}{ suspected severe + severe } \\
\hline gestation $<41$ weeks & & & $68: 532$ \\
\hline gest & $13: 2$ & $12: 234$ & $13: 231$ \\
\hline Asphyxia (all cases) & $34: 883$ & $32: 836$ & $32: 832$ \\
\hline gestation $<41$ weeks & $23: 632$ & $21: 596$ & $21: 595$ \\
\hline $\mathrm{n} \geq 41$ weeks & $11: 251$ & $11: 240$ & $11: 237$ \\
\hline Morbidity (all cases) & $293: 624$ & $275: 593$ & $274: 590$ \\
\hline gestation $<41$ weeks & $222: 433$ & $206: 411$ & $209: 407$ \\
\hline $1 \geq 41$ weeks & $71: 191$ & $69: 182$ & $65: 183$ \\
\hline Admission to SCBU (all cases) & $183: 731$ & $171: 694$ & $169: 692$ \\
\hline gestation $<41$ weeks & $146: 507$ & $136: 479$ & $135: 479$ \\
\hline gestation $\geq 41$ weeks & $37: 224$ & $35: 215$ & $34: 213$ \\
\hline
\end{tabular}

Numerator $=$ number of cases with morbidity; denominator $=$ number of cases in group without morbidity.

SCBU-Special Care Baby Unit

sent" or "absent" are shown in Figure 2. The 95\% confidence intervals of these differences are indicated by error bars. The polarity of the error bar has been adjusted in each case so that a positive excursion reflects increased morbidity at the "diabetic" end of the spectrum of the index of maternal glucose handling, and the scale chosen is in proportion to the SD of the mean of the maternal index concerned. It can be seen that FPG and insulin response failed to predict morbidity in the infant. When the other indices (i.e. those which describe glucose disposal during the IVGTT) were examined, there was on average poorer glucose disposal among the "affected" group in almost all the morbidity categories examined, although only in some instances did this reach statistical significance. Summed plasma glucose (reflecting the area under the curve) appeared to be more strongly associated with morbidity in the infant than either II or AI.

Figure 3 illustrates the relationship between congenital abnormality and summed plasma glucose. The association extends across the entire range of summed plasma glucose, and in common with all the other relationships examined is not restricted to a discrete "pathological" group at the "diabetic" end of the normal spectrum.

The associations between maternal glucose metabolism and neonatal nutritional status and morbidity were also examined in subgroups defined on the basis of maternal weight, age, parity and smoking habit; no 


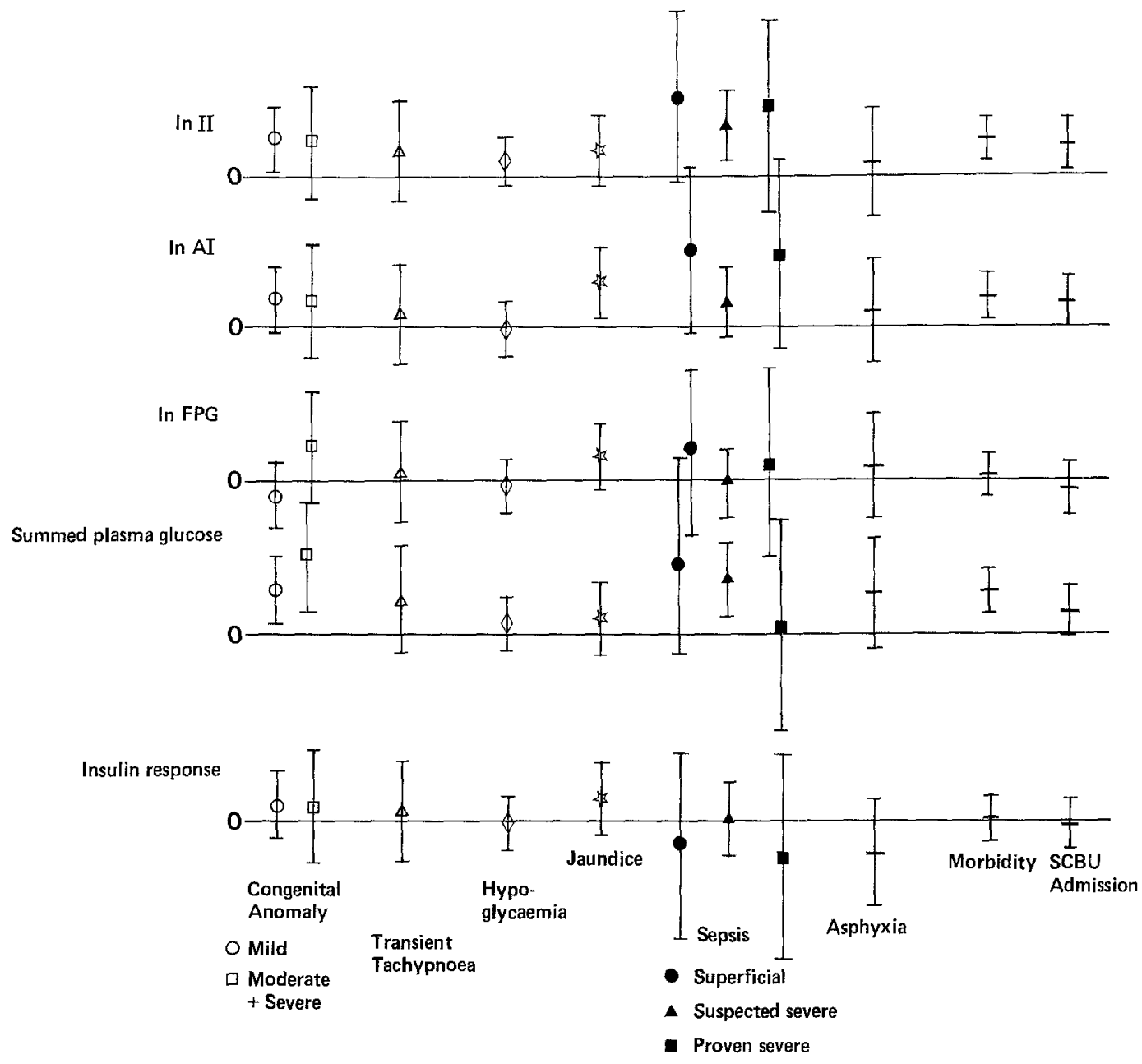

Fig. 2. Ninety-five percent confidence intervals for the differences in means between "pathological" and "normal" groups. When the symbol lies above the horizontal line, the mean maternal index for that neonatal morbidity group lies towards the diabetic end of the spectrum, and when the entire error bar lies above the line, the mean is significantly different from that of cases not in that particular morbidity group (see text for detailed explanation). The numbers of cases in the various groups are given in Table 2. "Hypoglycaemia" = plasma glucose $<2 \mathrm{mmol} / 1$

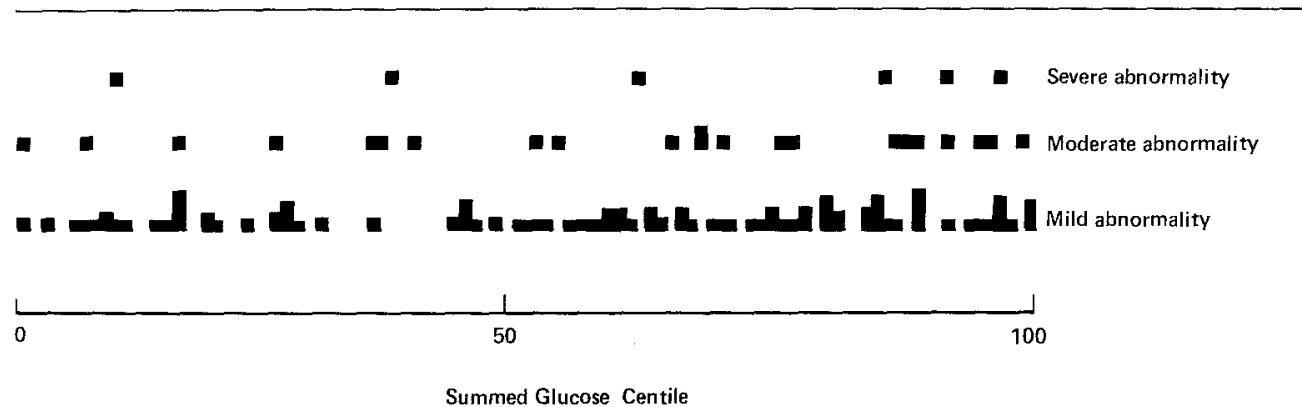

Fig. 3. Relationship between centile for maternal summed glucose and occurrence of malformations

consistent changes in the pattern of association emerged. However, when the study population was subdivided by gestational age at delivery, it became evident that the relationships between maternal glucose metabolism and certain forms of neonatal morbidity, particularly asphyxia and the need for admission to the special care baby unit, were enhanced at gestations in excess of 41 completed weeks (287 days) (Fig.4). The numbers of cases in the various groups illustrated in Figures 2 and 4 are shown in Table 2.

Details of cases where the infant died or suffered major handicap are shown in Table 3.

The distributions in the study population of the indices of maternal carbohydrate metabolism are shown in Figure 5. The interrelationships between these variables, and their relationships with maternal height, mid-pregnancy weight, age, social class, parity and gestation at the time of testing are presented as Pearson correlation coefficients in Tables 4 and 5.

\section{Discussion}

\section{Distribution of indices of maternal glucose metabolism}

The study population resembled the total hospital population in the variables examined; the results can therefore be used as standards for pregnant Caucasian women.

Parity had no apparent influence on maternal glucose disposal (II and Al) (Table 5), but the indices of maternal glucose metabolism did tend to deteriorate 
Table 3. Cases of fatal and serious morbidity

\begin{tabular}{|c|c|c|c|c|c|c|}
\hline $\begin{array}{l}\text { Clinical } \\
\text { diagnosis }\end{array}$ & FPG & AI & II & $\begin{array}{l}\text { Summed } \\
\text { glucose }\end{array}$ & $\begin{array}{l}\text { Insulin } \\
\text { response }\end{array}$ & Outcome \\
\hline \multicolumn{7}{|c|}{ 1. Massive placental infarct } \\
\hline & 3.9 & 2.89 & 5.78 & 47.3 & 217 & Stillbirth \\
\hline \multicolumn{7}{|c|}{ 2. Trisomy 18} \\
\hline & 4.3 & $1.33^{\mathrm{c}}$ & $2.43^{c}$ & $64.9^{\mathrm{c}}$ & $20^{\mathrm{c}}$ & Stillbirth \\
\hline \multicolumn{7}{|c|}{ 3. Severe birth asphyxia } \\
\hline & 4.7 & 2.52 & 8.42 & $61.5^{\mathrm{a}}$ & 166 & Died $<1 \mathrm{~h}$ \\
\hline \multicolumn{7}{|c|}{ 4. Rhizomelic chondrodysplasia punctata } \\
\hline & 4.4 & 2.04 & 4.21 & 52.4 & 139 & Died $<1 \mathrm{~h}$ \\
\hline \multicolumn{7}{|c|}{ 5. Arthrogryposis congenita multiplex } \\
\hline & 4.3 & 2.31 & 5.55 & 52.4 & $20^{c}$ & Died $<2$ years \\
\hline \multicolumn{7}{|c|}{ 6. Trisomy 21} \\
\hline & $5.1^{\mathrm{b}}$ & 2.07 & 4.62 & 53.2 & 75 & Survived \\
\hline \multicolumn{7}{|c|}{ 7. Meningo-encephalocele } \\
\hline & 4.1 & 2.31 & 4.47 & 56.5 & 93 & Survived \\
\hline \multicolumn{7}{|c|}{ 8. Idiopathic hyperinsulinism with intractable hypoglycaemia } \\
\hline & 4.2 & 2.61 & 5.78 & 43.5 & 152 & $\begin{array}{l}\text { Severely } \\
\text { retarded }\end{array}$ \\
\hline \multicolumn{7}{|c|}{ 9. Hyaline membrane disease at 38 weeks gestation } \\
\hline & 4.3 & 2.13 & 5.12 & 48.6 & 44 & Survived \\
\hline \multicolumn{7}{|c|}{ 10. Hyaline membrane disease at 39 weeks gestation } \\
\hline & 4.1 & $1.57^{\mathrm{a}}$ & $3.08^{\mathrm{a}}$ & 55.3 & 151 & Survived \\
\hline
\end{tabular}

a in "diabetic" 10 th centile; ${ }^{b}$ in "diabetic" 5 th centile; " in "diabetic" 2.5 th centile

Table 4. Pearson correlation coefficients $(r)$ between indices of maternal glucose metabolism. $n=$ number of cases available for analysis

\begin{tabular}{llcccc}
\hline & & $\begin{array}{l}\text { Insulin } \\
\text { response }\end{array}$ & $\begin{array}{l}\text { Summed } \\
\text { glucose }\end{array}$ & $1_{\mathrm{n}} \mathrm{AI}$ & \multicolumn{1}{l}{$\mathrm{l}_{\mathrm{n}} \mathrm{II}$} \\
\hline $\mathrm{l}_{\mathrm{n}} \mathrm{FPG}$ & $r$ & -0.0713 & 0.3705 & -0.1799 & 0.0449 \\
& $(n)$ & $(864)$ & $(868)$ & $(917)$ & $(917)$ \\
& $p$ & 0.018 & $<0.001$ & $<0.001$ & 0.087 \\
$\mathrm{l}_{\mathrm{n}} \mathrm{II}$ & $r$ & 0.2200 & -0.7415 & 0.7835 & \\
& $(n)$ & $(864)$ & $(868)$ & $(917)$ & \\
& $p$ & $<0.001$ & $<0.011$ & $<0.001$ & \\
$\mathrm{l}_{\mathrm{n}} \mathrm{AI}$ & $r$ & 0.2085 & -0.5734 & & \\
& $(n)$ & $(864)$ & $(868)$ & & \\
& $p$ & $<0.001$ & $<0.001$ & & \\
Summed & $r$ & -0.2450 & & & \\
glucose & $(n)$ & $(819)$ & & & \\
& $p$ & $<0.001$ & & & \\
\hline
\end{tabular}

with advancing maternal age. These relationships persisted when partial correlation coefficients were examined and are in keeping with existing knowledge $[6$, $20,21]$.

Whereas O'Sullivan and his coworkers $[20,21]$ reported that fasting blood glucose and the individual glucose values of the oral glucose tolerance test were distributed symmetrically, in the present series the distributions of FPG, AI and II were appreciably skewed (Fig. 5); centiles are therefore more appropriate in defining a "normal" range than are mean and standard deviation, and Student's t-tests and confidence intervals involving these indices have been calculated using their natural logarithms which produce more normal
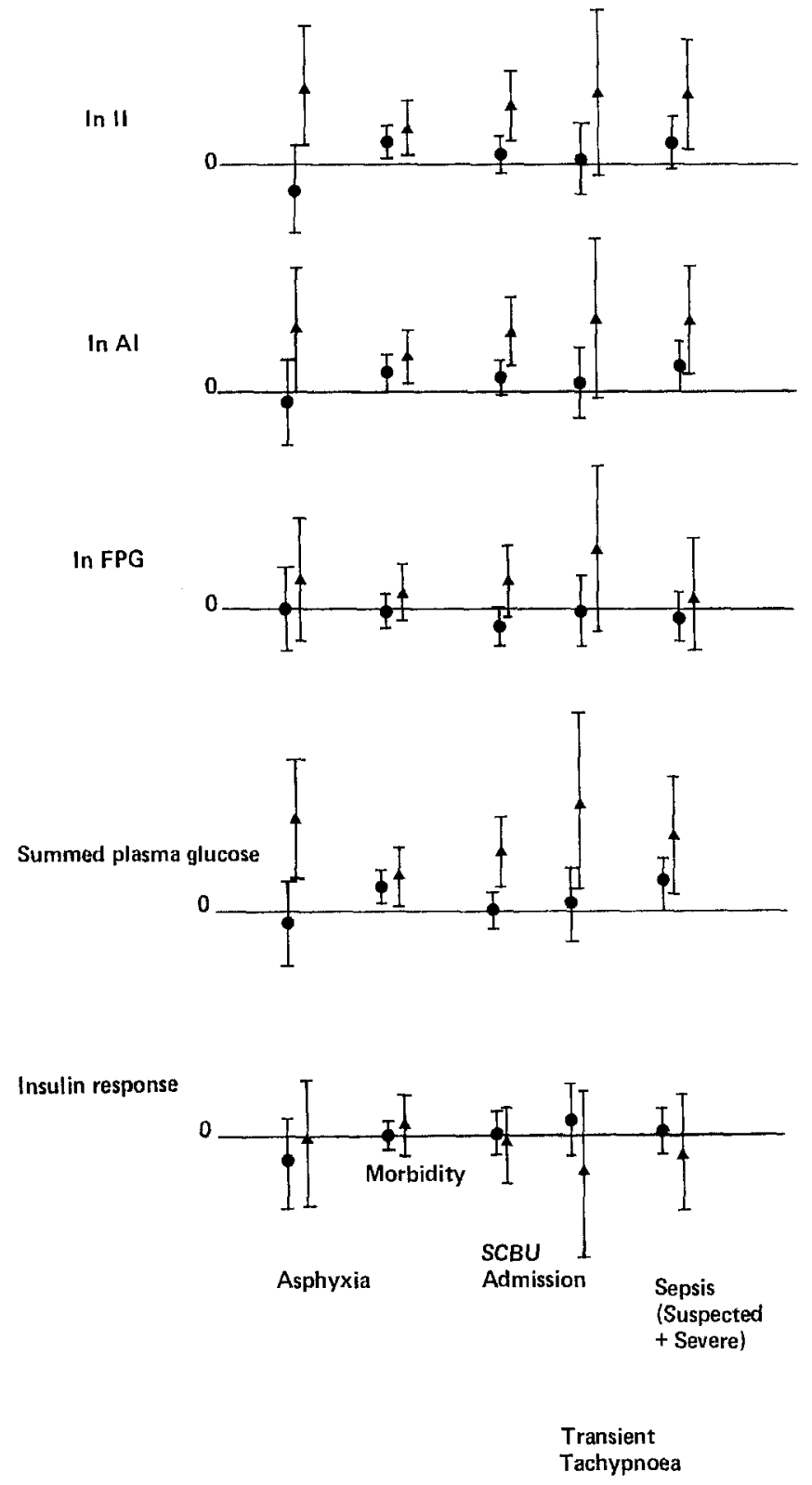

Fig. 4. Effects of gestational age at delivery on $95 \%$ confidence intervals (see legend for Fig. 2 and text for detailed explanation). The numbers of cases in the various groups are given in Table 2. Gestation $<41$ weeks, $\Delta$ Gestation $\geqslant 41$ weeks

distributions. Solomons et al. [5] stated that $2.5 \%$ of AI results in the third trimester of pregnancy should lie below 1.13; Billis and Rastogi [3] quoted a similar value of 1.17. These figures differ substantially from the 2.5 th centile value for AI obtained in the present study (1.41), reflecting the application by the previous authors of parametric statistics to the log transformed AI rather than the use of observed centile values.

With the exception of insulin response, the indices of maternal glucose metabolism were distributed unimodally (Fig. 5), with no evidence of a discrete "pathological" group towards the diabetic end of the distributions. In this respect the distributions are similar to 


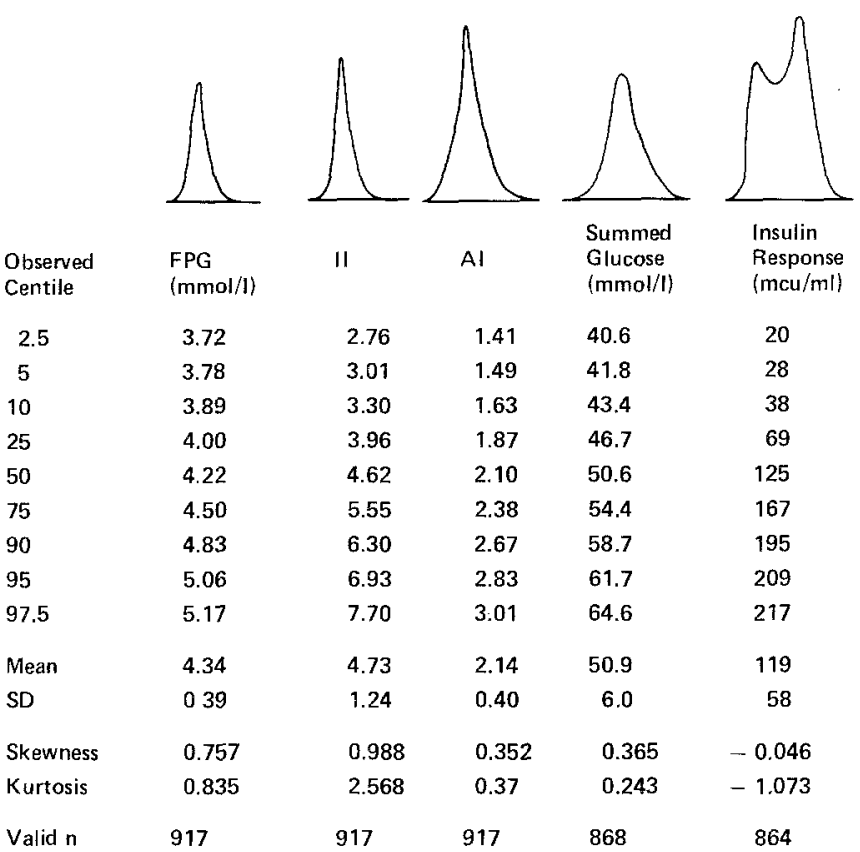

Fig.5. Distributions of maternal fasting plasma glucose (FPG), increment index (II), absolute index (AI), summed plasma glucose and insulin response to injected glucose $(n=$ number of cases available for analysis)

Table 5. Pearson correlation coefficients $(r)$ between indices of maternal glucose metabolism, logged where appropriate $\left(l_{n}\right)$, and various maternal characteristics. ( $n=$ number of cases available for analysis)

\begin{tabular}{lllllll}
\hline $\begin{array}{l}\text { Maternal } \\
\text { characteristic }\end{array}$ & $\mathrm{l}_{\mathrm{n}} \mathrm{FPG}$ & $\mathrm{l}_{\mathrm{n}} \mathrm{II}$ & $\mathrm{l}_{\mathrm{n}} \mathrm{AI}$ & $\begin{array}{l}\text { Summed } \\
\text { glucose }\end{array}$ & $\begin{array}{l}\text { Insulin } \\
\text { response }\end{array}$ \\
\hline Height & $r$ & 0.0685 & 0.0966 & -0.0585 & -0.2080 & -0.0662 \\
& $(n)$ & $(916)$ & $(916)$ & $(916)$ & $(867)$ & $(863)$ \\
& $p$ & 0.019 & 0.002 & 0.039 & $<0.001$ & 0.026 \\
Weight & $r$ & 0.1832 & 0.0110 & -0.2072 & -0.1311 & 0.0918 \\
mid- & $(n)$ & $(787)$ & $(787)$ & $(787)$ & $(748)$ & $(745)$ \\
pregnancy & $p$ & $<0.001$ & 0.379 & $<0.001$ & $<0.001$ & 0.006 \\
Social & $r$ & 0.0187 & 0.0577 & 0.0580 & -0.0484 & 0.1525 \\
class & $(n)$ & $(876)$ & $(876)$ & $(876)$ & $(828)$ & $(827)$ \\
& $p$ & 0.290 & 0.044 & 0.043 & 0.082 & $<0.001$ \\
Age & $r$ & 0.1076 & -0.1063 & -0.1463 & 0.0702 & -0.1905 \\
& $(n)$ & $(917)$ & $(917)$ & $(917)$ & $(868)$ & $(864)$ \\
Parity & $p$ & 0.001 & 0.001 & $<0.001$ & 0.019 & $<0.001$ \\
& $r$ & 0.0557 & -0.0062 & -0.0376 & -0.0040 & -0.0505 \\
Gestation & $(n)$ & $(917)$ & $(917)$ & $(917)$ & $(868)$ & $(864)$ \\
at & $p$ & 0.046 & 0.426 & 0.128 & 0.453 & 0.068 \\
testing & $(n)$ & $(916)$ & $(916)$ & $(916)$ & $(867)$ & $(863)$ \\
\hline
\end{tabular}

those previously reported for the oral glucose tolerance test $[20,21]$.

\section{Relationships between indices of maternal glucose metabolism}

From a purely mathematical point of view, it makes little difference in interpreting the intravenous glucose tolerance test whether the FPG is subtracted before the disappearance rate is calculated (II), or the absolute glucose value is used (AI), because neither of these approaches produces a true exponential decay. Despite differences in how the two indices (AI and II) related to fetal nutrition (Table 1) and morbidity (Fig. 2), we have no reason to suggest that one index reflects maternal glucose disposal more accurately than the other. Summed glucose, which approximates the area under the glucose disappearance curve, correlated with both the FPG and the indices of glucose disappearance, and correlated well with neonatal morbidity (Figs.2-4). This index offers advantages to the clinician in giving equal weight to each point on the disappearance slope, in ease of calculation and in the absence of the bias in interpretation which is inherent in drawing "best-fit" lines for the calculation of II and AI.

The poor correlations between maternal FPG, insulin response and indices of glucose disposal suggest that it is inappropriate to screen for glucose intolerance during pregnancy using the FPG or the insulin response to injected glucose.

\section{Relationships between maternal indices and neonatal nutrition}

Glucose intolerance during pregnancy leads to increased birthweight relative to gestation [1], and even in nondiabetic mothers delayed glucose disposal is associated with an increased incidence of neonatal macrosomia [2]. In this study, variations in maternal glucose tolerance within the normal range played a weak but highly significant role in the determination of longitudinal size and adiposity in the newborn infant (Table 1). The strongest of these associations, that between AI and corrected birthweight, is shown graphically in Figure 1.

It can be seen from Table 1 that whereas diminished maternal glucose disposal, particularly AI, correlated strongly with all the neonatal measurements, FPG in contrast correlated strongly with skinfold thickness but much less strongly with the other neonatal measurements. The relatively poor correlations already noted between FPG and the indices of maternal glucose disposal (particularly II which is calculated independently of FPG) suggest that FPG in nondiabetic pregnant women is controlled by mechanisms which differ from those controlling glucose disposal. Variations in maternal FPG and glucose disposal also appear to have different effects on fetal growth and development. It is therefore speculated that the fetus exposed to the prolonged stimulus of a relatively high maternal FPG maintains glucose homeostasis by diverting glucose to lipogenesis to a greater extent than does the fetus exposed to the more variable hyperglycaemia likely to result from maternal glucose intolerance who appears to maintain euglycaemia by diverting glucose to a wider variety of growth-promoting anabolic processes. 


\section{Relationships between maternal indices and neonatal morbidity}

Glucose intolerance during pregnancy is associated with an increased incidence of congenital malformations and other neonatal morbidity [1]. In the present study AI, II and summed glucose were significantly associated with morbidity, and the patterns of morbidity were similar in each instance (see Figs. 2 and 3).

Asphyxia and asphyxia-related morbidity. In addition to those infants who fulfilled our criteria for neonatal asphyxia, other diagnostic categories probably included a high proportion of asphyxiated infants. In particular, the maternal indices were significantly associated with the clinical suspicion of sepsis and not with bacteriologically proven sepsis. In defining "suspected sepsis" we included all babies who had been given antibiotics, and experience indicates that perinatal asphyxia, recognised or occult, plays a major part in determining the administration of antibiotics, reflecting the antibiotic policy of the neonatologists responsible for the clinical care of the infants, who were independent of the workers involved in this research and had no knowledge of the results of the maternal tests. Suspected perinatal asphyxia is similarly a major determinant of admission to the special care baby unit.

In the study population as a whole, the indices of maternal glucose metabolism did not influence significantly the incidence of asphyxia or asphyxia-related problems (Fig. 2), but in infants who were post-term a clear relationship emerged between maternal glucose intolerance and asphyxia-related problems (Fig.4).

The incidence of perinatal asphyxia is known to be increased not only in the infants of Type 1 (insulin-dependent) diabetic mothers but also in the infants of mothers with gestational diabetes [22], a phenomenon which may reflect the stimulant effect of increased substrate availability on fetal oxidative metabolism [23]. The results of the present study suggest that changes in maternal glucose tolerance, insufficiently severe to justify a diagnosis of diabetes, may enhance the risk of perinatal asphyxia if pregnancy proceeds beyond term.

Other morbidity. In the 9 mothers whose offspring suffered fatal or serious morbidity or non-hereditary malformation (see Table 3), there was an unexpectedly high proportion of indices of glucose metabolism in the tenth centile towards the "diabetic" end of the normal range.

In Case 2 (trisomy 18), there was convincing evidence of poor glucose disposal with a poor insulin response and in Case 6 (trisomy 21) FPG was above the 95th centile. Although maternal glucose intolerance has not been described previously in association with trisomy 18 it has been associated with other chromosomal disorders [24-26] and the offspring of diabetic mice show an increased incidence of chromosomal abnormality [27]. In these two instances the occurrence of autosomal trisomies in the offspring of otherwise asymptomatic mothers might have been related to impaired glucose metabolism.

In Case 8, a mother with completely normal indices of glucose metabolism produced the only baby in the study population with severe clinical disturbance of carbohydrate metabolism, details of which have been reported elsewhere [28].

\section{Predictive value of FPG and maternal glucose tolerance}

Although variations in maternal glucose metabolism within the normal range appeared to play a significant biological role in the determination of fetal size and in the pathogenesis of congenital abnormalities and neonatal morbidity (particularly morbidity related to perinatal asphyxia), there was no uniformity of abnormality within the extreme centiles; the degree of risk to the baby was graduated throughout the normal range of maternal glucose tolerance. We examined large numbers of computer-generated scatter diagrams showing relationships between indices of maternal glucose metabolism and neonatal morbidity; all resembled Figures 1 and 3 in that there was no single level of maternal glucose intolerance in the nondiabetic population beyond which fetal morbidity increased sharply, or below which enhanced fetal well-being was assured.

Similar results were obtained in the smaller series of Tallarigo et al. [2] in which there appeared to be a continuous increase in the incidence of neonatal macrosomia and of congenital malformation with diminishing maternal glucose tolerance. Thus, in common with the oral glucose tolerance test, the intravenous glucose tolerance test is "not suited to diagnose fetal disease or to exclude it with certainty" [29] in the individual case.

Surprisingly, the maternal insulin response to the intravenous injection of glucose, which is known to be a sensitive indicator of diminishing pancreatic B-cell function in non-pregnant subjects [15], correlated with the indices of neonatal nutrition and morbidity much less frequently than the other indices of maternal glucose tolerance (Figs.2 and 4). Although insulin response correlated significantly with the other maternal biochemical indices (Table 4), the magnitude of the correlations was such as to suggest that insulin output plays a relatively small part in determining glucose tolerance in non-diabetic pregnancy.

\section{Practical implications}

Our results indicate that variations within the normal range in maternal glucose tolerance measured at 32 weeks gestation correlate with the incidence of nonchromosomal and perhaps chromosomal congenital anomalies. It is reasonable to speculate that these associations would also have been present if maternal glucose tolerance had been measured at the time of germ-cell division or organogenesis, and that congeni- 
tal anomaly is a possible consequence of glucose tolerance towards the diabetic end of the normal spectrum. This speculation is supported by the observations of Widness et al. [1] and Tallarigo et al. [2] who found significant relationships between maternal glucose tolerance and the incidence of congenital malformations in the offspring of nondiabetic mothers, and of Fuhrmann et al. [30] who reported that extremely strict control of maternal diabetes, starting prior to conception, reduced the malformation rate in the offspring of diabetic mothers to less than that observed in a control nondiabetic population.

Thus, although the intravenous glucose tolerance test lacks predictive sensitivity in individual cases and cannot be used to identify a small pathological or highrisk group of mothers, we believe that our findings have practical implications; it can be deduced from Figure 3 that if glucose handling before conception were improved over the entire population, a change which might be effected by diet without recourse to drugs [31], the total burden of congenital malformations might be reduced, and from Figure 4 that when maternal glucose disposal is known to be reduced, pregnancy should not be allowed to proceed beyond term because of the increased risk of perinatal asphyxia and its complications.

Acknowledgements. This work was supported by grants from the University of Aberdeen, the Secretary of State for Scotland (Grant Ref 85.8750) and the Wellcome Foundation. We wish to thank Professor I.A. MacGillivray, Professor A.G.M. Campbell and the Senior Obstetrical and Paediatric Staff, Aberdeen Maternity Hospital for the cooperation which made this study possible. We wish particularly to acknowledge the leadership and active participation over many years of Emeritus Professor J.M. Stowers. Ms. F. MacGregor was responsible for most of the capillary sampling and glucose analyses.

\section{References}

1. Widness JA, Cowett RM, Coustan DR, Carpenter MW, Oh W (1985) Neonatal morbidities in infants of mothers with glucose intolerance in pregnancy. Diabetes 34 [Suppl 2]: 61-65

2. Tallarigo L, Giampietro O, Penno G, Miccoli R, Gregori G, Navalesi R (1986) Relation of glucose tolerance to complications of pregnancy in nondiabetic women. N Engl J Med 315: 989-992

3. Billis A, Rastogi GK (1966) Studies in methods of investigating carbohydrate. Diabetologia 2: 169-177

4. Butterfield WJH, Abrams ME, Whichelow MJ (1971) The 25-g intravenous glucose tolerance test: a critical appraisal. Metabolism 20: 255-265

5. Solomons E, Silverstone FA, Posner NA (1963) Obstetric factors suggesting diabetes evaluated by the rapid intravenous glucose tolerance test. Obstet Gynecol 22: 50-55

6. Lunell N-O (1966) Intravenous glucose tolerance in women with previously complicated pregnancies. Acta Obstet Gynecol Scand 45 [Suppl 4]: 1-89

7. O'Sullivan JB, Snyder PJ, Sporer AC, Dandrow RV, Charles D (1970) Intravenous glucose tolerance test and its modification by pregnancy. J Clin Endocrinol Metab 31: 33-37

8. Hadden DR, Harley JMG, Kajtar TJ, Montgomery DAD (1971) A prospective study of three tests of glucose tolerance in pregnant women selected for potential diabetes with reference to foetal outcome. Diabetologia 7: 87-93
9. Silcock DH, Hadden DR, Neill DW (1972) Computer analysis of intravenous glucose tolerance tests. Diabetologia 8: 301-304

10. Fisher PM, Hamilton PM, Sutherland HW, Stowers JM (1974) The effect of pregnancy on intravenous glucose tolerance. J Obstet Gynaecol Br Commonw 81: 285-290

11. Feige A, Mitzkat H-J, Zick R, Jakobitz K (1984) Untersuchungen zum Einfluß der Schwangerschaft auf den Kohlenhydrat- und Fettstoffwechsel der Mutter. Z Geburtshilfe Perinatol 188: $167-173$

12. Hinckers HJ (1977) Glukose-Stoffwechsel in der Schwangerschaft. Urban \& Schwarzenberg, München Wien Baltimore

13. Office of Population Censuses and Surveys (1970) Classification of Occupations. Her Majesty's Stationen Office, London

14. Duncan LJP (1956) The intravenous glucose tolerance test. Q J Exp Physiol 41: 85-96

15. Srikanta S, Ganda OP, Gleason RE, et al. (1984) Pre-type 1 diabetes. Linear loss of beta cell response to intravenous glucose. Diabetes 33: 717-720

16. Altman DG, Coles EC (1980) Assessing birth weight-for-dates on a continuous scale. Ann Hum Biol 7: 35-44

17. Farmer G (1985) Neonatal skinfold thickness. Measurement and interpretation at or near term. Arch Dis Child 60: 840-842

18. SPSS Inc, Illinois (1986) SPSSx User's Guide, 2nd edn. McGraw Hill, New York

19. Gardner MJ, Altman DG (1986) Confidence intervals rather than $\mathrm{P}$ values: estimation rather than hypothesis testing. $\mathrm{Br}$ Med J 292: 746-750

20. Wilkerson HLC, O'Sullivan JB (1963) A study of glucose tolerance and screening criteria in 752 unselected pregnancied. Diabetes 12: $313-318$

21. O'Sullivan JB, Mahan CM (1964) Criteria for the oral glucose tolerance test in pregnancy. Diabetes 13:278-285

22. Macfarlane CM, Tsakalakos N (1985) Evidence of hyperinsulinaemia and hypoxaemia in cord blood of neonates born to mothers with gestational diabetes. S Afr Med J 19: 81-84

23. Philipps AF, Rosenkrantz TS, Raye J (1985) Consequences of perturbations of fetal fuels in ovine pregnancy. Diabetes 34 [Suppl 2]: 32-35

24. Navarrette VN, Torres IH, Rivera IR, Shor VP, Gracia PM (1967) Maternal carbohydrate disorder and congenital malformations. Diabetes 16: 127-130

25. Milunsky A (1970) glucose intolerance in the parents of children with Down's syndrome. Am J Ment Defic 74: 475-478

26. Forbes AP, Engel E (1963) The high incidence of diabetes mellitus in 41 patients with gonadal dysgenesis, and their close relatives. Metabolism 12: 428-439

27. Endo A, Ingalls TH (1968) Chromosomal abnormalities in embryos of diabetic mice. Arch Environ Health 16: 316-325

28. Aynsley-Green A, Weindling AM, Soltesz G, Jenkins PA (1983) Transient lactic acidosis and hyperalaninaemia associated with neonatal hyperinsulinaemic hypoglycaemia: the effects of dichloroacetate (DCA). Eur J Pediatr 141: 114-117

29. Weiss PAM, Hofman H, Winter R, Purstner P, Lichtenegger W (1984) Gestational diabetes and screening during pregnancy. Obstet Gynecol 63: 776-780

30. Fuhrmann K, Reiher H, Semmler K, Fischer F, Fischer M, Glockner E (1983) Prevention of congenital malformations in infants of insulin-dependent diabetic mothers. Diabetes Care 6: 219-223

31. Sartor G, Schersten B, Carlstrom S, Melander A, Norden A Persson G (1980) Ten-year follow-up of subjects with impaired glucose tolerance. Prevention of diabetes by tolbutamide and diet regulation. Diabetes 29: 41-49

Received: 26 May 1987

and in revised form: 30 December 1987

Dr. G. Russell

Royal Aberdeen Children's Hospital

Cornhill Road

Aberdeen AB9 2ZG

UK 